\title{
Multicultural Working Teams and Safety Awareness: How Effective Leadership Can Motivate Safety Behaviour
}

\author{
Marianne Starren \\ Communication and Information Sciences, Radboud University, Nijmegen, The Netherlands \\ Email: m.starren@let.ru.nl
}

Received 5 May 2016; accepted 4 July 2016; published 7 July 2016

Copyright (C) 2016 by author and Scientific Research Publishing Inc.

This work is licensed under the Creative Commons Attribution International License (CC BY). http://creativecommons.org/licenses/by/4.0/

(c) (i) Open Access

\begin{abstract}
This empirical study focuses on the diversity of cultural values and leadership preferences in multicultural working teams and how this interaction makes it difficult to guarantee and motivate safety at the workfloor. It is often hypothesized that when employees from different national identities have to work together in one team, their different cultural backgrounds make a common mental state of safety awareness very complex. In managing this diversity of cultural values, the role of leadership style could be crucial. The results show that a cultural value as uncertainty avoidance clearly has its influence on safety awareness and that this outcome is mediated by the motivating leadership style.
\end{abstract}

\section{Keywords}

Multiculturalism, Safety at the Workfloor, Leadership Style and Motivation

\section{Introduction}

The need for new tools for managing the increasing diversity on multicultural workfloors in order to foster safety is growing. The results of several studies (see EU-OSH, 2003) have shown that employees in multicultural organisations are more affected by safety risks than in monocultural organisations. Moreover, several studies have revealed that migrant workers are more often involved in occupational accidents than national workers (EU-OSHA, 2013).

However, existing approaches to stimulate safe behaviour mostly fail to devote explicit attention to the variety of cultural backgrounds of their employees on the workfloor. Hale, Guldenmund, Van Loenhout, \& Oh (2010) refer to the serious consequences of diversity in relation to safety knowledge, (cultural) values about work and 
communication. In their study, it is shown that migrants are more vulnerable to safety incidents owing to aspects such as obedience (e.g. they are more reluctant to raise safety issues), language barriers, and eagerness to earn money quickly. For example, in the Netherlands it is expected that half of the migrants from Mid- and East European countries will enter via an employment agency (Ministry of Social Affairs and Employment in the Netherlands, 2011). Their more flexible (insecure) and so more vulnerable labour position will probably make the workers more reluctant to come up with safety issues. The most crucial factor, however, seems to be the lack of a shared safety climate because of different cultural backgrounds within the multicultural teams. It is important to provide more insight into how different cultural values of safety such as risk perception, safety motivation and insecurity feelings may influence the safety climate of a multicultural working team.

So, the need for approaches and tools that pay attention to language issues and aspects of cultural background for the prevention of accidents has grown. One of such tools could be the managing role of the leader in such diverse teams. His motivating safety style could be the key to create a common safety awareness in multicultural working teams. The Safety Specific Transformational Leadership style (SSTL) has been shown already effective to foster safety on the workfloor in previous studies (Barling, Weber, \& Kelloway, 1996; de Koster, Stam, \& Balk, 2011; Kelloway, Mullen, \& Francis, 2006). This leadership style is composed out of four different behaviour and communication styles: Idealized influence refers to acting as a role model for safety behaviour, Inspirational motivation means communicating a safety vision that encourages employees to behave accordingly, Intellectual stimulation makes employees think of "new ways of doing work" to improve safety and a transformational leader with Individualized consideration shows active interest and care for the personal safety of employees and individually supports them to achieve it (de Koster et al., 2011: p. 756). Depending on cultural values and the cultural dynamics within a multicultural team, one component or the other could be more effective to guarantee safety at the workfloor.

\section{Cultural Diversity and Safety at the Workfloor}

\subsection{Cultural Values at a Safe Workplace}

Workforce diversity refers to "the mix of people from various backgrounds in the labour force" (Saee, 2005: p. 41). People with different national backgrounds vary in attitudes, values and beliefs. It is therefore likely that members of multicultural work teams also vary in risk perception and perceptions of safety climate (EU-OHSA, 2013). When people with different cultural backgrounds are working together, there is a pool of different views and ideas which also brings certain benefits and drawbacks. It is argued that multicultural teams increase the amount of "ambiguity, complexity and confusion”, which may lead to miscommunication especially when it comes to finding consensus and trying to guide employees to think about certain procedures, e.g. safety, in the same way (Saee, 2005: p. 43). Creating a shared vision on safety climate is therefore crucial (EU-OSHA, 2013). A potential advantage of multicultural workforces is an increased “creativity and flexibility”, which can lead to new and innovative ideas (Saee, 2005: p. 43). Finding the balance between the advantage of new (other cultural) perspectives on safety issues on the workfloor and the disadvantage of lacking a shared safety climate, this study investigates the influence of one particular safety specific cultural value: uncertainty avoidance (Hofstede, 2011). A definition of a (national) culture that has been widely acknowledged is "the collective programming of the mind that distinguished the members of one group or category of people from another” (Hofstede, 2001: p. 9). Although many more different frameworks could be used for categorizing (national) cultural values (e.g. Trompenaars, 1993 and Schwartz, 1994; for an overview, see Soares et al., 2007), we focus in this study on Hofstede's framework of five different dimensions. We only use uncertainty avoidance as the most typically safety related variable dimension in our empirical analyses as we will explain in the following.

One of the first and few studies that examined if cultural values predict occupational injuries is the study by Reniers \& Gidron (2013). Using Hofstede's dimensions and investigating 22 European countries, they found that high power distance led to more injuries whereas high levels of individualism led to fewer injuries. Reniers \& Gidron (2013) found that both individualism and power distance could be influential under the same factor; the concept of "self-leadership", which according to them can lead to improved safety climate (Reniers \& Gidron, 2013: p. 76). Self-leadership involves: "responsibility, innovation, high self-esteem, motivation, selfknowledge” and emphasizes the role of "good communication” (Reniers \& Gidron, 2013: p. 79). According to this research, self-leadership is a "cultural concept", characterized by high individualism and low power distance (Reniers \& Gidron, 2013: p. 79). Reniers \& Gidron (2013) argue that self-leadership derives from transforma- 
tional leadership and in turn they predicted that both self-leadership and transformational leadership led to better safety results. They suggest trainings aimed at improving self-leadership by increasing individualism and lowering the level of power distance. However, because national culture with its beliefs and values can be seen as the "collective programming of the mind (...)" (Hofstede, 2001, see above), simply changing cultural values through training is therefore highly doubtable.

In their research, Mearns \& Yule (2008) found that high power distance was associated with one-way communication between managers and workers. Senior managers perceived to show more commitment to safety, e.g. through trustworthiness and general concern for employees' well-being, led to lower risk taking behaviour of the workforce. Mearns \& Yule (2008) argue that in high power distance cultures, frontline managers are less likely to use the knowledge of employees for the improvement of safety. In fact they found that perceived commitment of management to safety is a mediating factor to motivate employees in behaving safely even if their cultural beliefs might lead them to greater risk taking. Subsequently, Mearns \& Yule (2008) call for a "multi-level approach" that researches the impact of different cultural values and leadership style on safety outcomes and how in particular management commitment to safety can influence safety climate (Mearns \& Yule, 2008: p. 784). In the light of these results, the present study focuses on the role of uncertainty avoidance and leadershipstyle as potential predictor of a teams' safety climate by measuring safety awareness and the employees' perception of Safety Specific Transformational Leadership (Barling et al., 2002; de Koster et al., 2011; Kelloway et al., 2006). In previous research (e.g. Cultpepper \& Watts, 1988 and Dorfman \& Howell, 1999) it has often been tried to use Hofstede's cultural dimensions on an individual level instead of at the national level. One shortcoming for the scales suggested in the Dorfman and Howell (1999) study was confirmed in a previous version of this study, namely a marginal alpha coefficient (.55) for the power distance scale: "It is not clear why this should be the case in that items appear to possess reasonable content validity and the principal components analysis (Dorfman \& Howell, 1999, p. 30)

\subsection{Safety Behaviour and Leadership}

Several studies have indicated that leadership indeed plays an important role to enhance safety in multicultural teams (Saee, 2005; EU-OSHA, 2013; Kearney \& Gebert, 2009). In order to be effective in managing cultural diversity, a leader has to be actively engaged to use the potential inherent in multicultural teams, while being sensitive to cultural issues (Saee, 2005: p. 44). According to the European Agency of Occupational Health and Safety (2013), transformational leadership SSTL is an effective management approach to cultural diversity because it supports an "inclusive organization", one that exploits the potential of diversity and creates a shared vision within the diverse team (EU-OSHA, 2013: p. 7). Interestingly, the same leadership aspects exploiting the positive potential in workforce diversity are also positively related to better occupational health and safety (EU-OSHA, 2013). As a conclusion, transformational leadership is supposed to be a management style that is effective in enhancing occupational safety in general but especially effective in multicultural teams, as it creates a shared vision through letting employees think actively about issues and taking into account their different ideas (EU-OSHA, 2013). Kearney \& Gebert (2009) studied 62 R\&D teams and examined SSTL as a moderator for differences in workforce in nationality, education, age and team outcomes (Kearney \& Gebert, 2009). They found that SSTL can help to use the positive potential inherent in multicultural teams for their safety performance. When SSTL was high, nationality was positively related to team performance (Kearney \& Gebert, 2009). Again, a reason for that might be that SSTL enhances knowledge exchange and the group's identification with a collective vision (Kearney \& Gebert, 2009). Kearney and Gebert did not fully examine if and how SSTL can help using the positive potential of a culturally diverse workforce while balancing out the negative aspects but they hypothesize that SSTL has this "dual effect” (Kearney \& Gebert, 2009: p. 80).

Therefore they call for more research to examine under which conditions SSTL is most effective and to take into account the perceived SSTL level of the leader by the employees. Interestingly, in their study, leaders viewed themselves more SSTL than their followers actually perceived them (Kearney \& Gebert, 2009). The present study therefore examines the perceptions employees have of their leader's style and the role this might play in predicting safety climate.

Other studies have found that there are differences in preferred leadership style among different cultures (e.g. Saee, 2005). Therefore transformational leadership may not necessarily be effective in all contexts. In his book, Saee (2005) makes suggestions based on a wide range of research literature on issues concerning the global 
economy and organizational management. He argues that even if the requirements on leaders to motivate and make decisions are universal (Saee, 2005), there are different cultural preferences in leadership due to the differences in distributed authority and responsibility among societies. According to Saee (2005), employees from high power distance cultures are used to hierarchical power distribution in which every worker has a specific role and are uncomfortable with leaders that delegate important decisions to their employees.

According to Saee (2005), high uncertainty avoidance cultures see uncertainty and ambiguity as a threat and are worried about future situations. Therefore, they rely on leaders that provide employees with clear task instructions and rules and may not feel comfortable with participating in important decision-making processes (Saee, 2005). While the present study focuses on perceived leadership styles and their predictive role for safety climate, future research should also take into account the role of cultural values as predictors for employees' preferred leadership style.

The research by Barling, Loughlin, \& Kelloway (2002) is one of the first studies that examined Safety Specific Transformational Leadership (SSTL) and its effects on safety (Barling et al., 2002). Barling et al. (2002) identified SSTL as a predictor of safety climate. Through these mediating variable, SSTL also indirectly influenced safety results (Barling et al., 2002). Kelloway, Mullen, \& Francis (2006) build on Barling et al. (2002) and examined the effects of passive and active leadership style on safety outcomes. They found that passive and active SSTL had opposite effects on safety climate and safety consciousness. They argue that there is no "neutral position" when it comes to managing safety (Kelloway et al., 2006: p. 83). Leadership behaviour always had either positive or negative effects on injury rates (Kelloway et al., 2006). They furthermore hypothesize that SSTL could have a totally different effect. Employees could become too focused on productivity and to "exceed the expectations" that they might ignore safety rules (Kelloway et al., 2006: p. 85). This study was furthermore conducted in different organizations. Differences could therefore also be the consequence of organizational differences.

de Koster et al. (2011) identified factors that influence the number of incidents that occurred in 78 Dutch warehouses. They found that SSTL and safety awareness are potential factors that influence safety performance. According to de Koster et al. (2011), leadership is crucial in developing safety because they found that SSTL had a direct influence on safety performance. SSTL is considered a "key driver of safety performance" because leadership will on the one hand predict that Hazard Reducing Systems are implemented and SSTL increases safety awareness directly (de Koster et al., 2011: p. 754). Furthermore, SSTL is ultimately improving safety performance through its positive influence on safety awareness. de Koster et al. (2011) call for more research that investigates the factor safety climate in greater detail (de Koster et al., 2011). The role of communication style in leadership is again prevalent. Several studies have identified different communicative characteristics of the safety-specific transformational leader (e.g. Barling et al., 2002; de Koster et al., 2011; Kelloway et al., 2006). Dubbel "Idealized influence" refers to acting as a role model for safety behaviour, "Inspirational motivation" means communicating a safety vision that encourages employees to behave accordingly, "Intellectual stimulation" makes employees think of "new ways of doing work" to improve safety and a transformational leader with "Individualized consideration" shows active interest and care for the personal safety of employees and individually supports them to achieve it (de Koster et al., 2011: p. 756). This study also aims to identify the influences these dimensions might have individually on safety climate, to give managers a practical guideline which leadership characteristics in particular can enhance workplace safety.

The present study aims to respond to the gaps in cross-cultural research and practice in safety at the workfloor, through investigating which factors play a predictive role for a better safety climate in multicultural work teams. The investigation of how cultural differences in uncertainty avoidance play a role in predicting safety climate, as well as being able to assess if an employees' perception of their leader's safety specific transformational predicts safety climate, poses valuable knowledge for managers and safety research organizations that want to create safety guidelines and programs for a culturally diverse workforce.

\section{An Empirical Study on the Role of Leadership and Cultural Values in Fostering Safety in a Multicultural Working Environment}

The present study aims to investigate the role of cultural value differences (e.g. uncertainty avoidance) and safety specific leadership style (SSTL) as potential predictors for safety climate/safety awareness. As in the study of de Koster et al. (2011) we distributed a questionnaire in 13 multinational companies in the warehouse 
sector in the Netherlands (total number of participants was 224). Most warehouses used in this study were located around the city of Nijmegen, and/or the eastern part of the Netherlands.

\subsection{Method, Instruments and Definitions}

Safety Awareness. Barling et al. (2002) and de Koster et al. (2011) define safety awareness as an "individual's own awareness of safety issues" and "being mentally aware of safety in your work and knowing what behaviours foster operational safety” (de Koster et al., 2011: p. 755). Safety awareness was measured using de Koster et al.'s (2011) questionnaire items modified by seven 7-point Likert scales, originally developed by Barling et al. (2002). Respondents could indicate their agreement or disagreement with a statement (e.g. "I am well aware of the safety risks involved in my job"), choosing a point on the scale between 1 "I absolutely disagree" and 7 "I absolutely agree". Cronbach's alpha was good for safety awareness $(\alpha=.87)$.

Uncertainty Avoidance. Hofstede's work on cultural dimensions has been one of the most cited theoretical frameworks in the area of international cross cultural studies to examine the different hierarchies of values across cultures. However, Hofstede's work focuses on the societal and national level of culture. Therefore, in the present study we focus on individual values, using the suggested scales by Culpepper \& Watts (1999) for uncertainty avoidance (UA), which were originally developed by Dorfman and Howell (1988) on the basis of Hofstede's original scales. Culpepper and Watts tested these scales and found that they indeed are a promising tool to measure individual cultural values and to link them easier to organizational communication aspects such as job satisfaction and commitment (Culpepper \& Watts, 1999).

Uncertainty avoidance is measured using the scales as recommended by Dorfman and Howell (1988), with five items for uncertainty avoidance. Respondents were asked to rate statements such as "Managers expect employees to closely follow instructions" by choosing a point on a 7-point scale between 1 "I totally disagree" and 7 "I totally agree". The scales for uncertainty avoidance were reliable, Cronbach's alpha was good $\alpha=.90$.

Leadership style. Transformational leadership can be viewed as the "developed" leadership variant and especially safety-specific transformational leadership (SSTL) is positively related to safety climate as it refers to the topic specifically (EU-OSHA, 2013). According to de Koster et al. (2011: p. 756) transformational leadership "inspires employees to go beyond their self-interest", to focus more on shared values of the organization and therefore to "perform beyond expectations". de Koster et al. (2011) further define SSTL as a "highly effective leadership style, concerned about the well-being of the employees" that has a positive influence on "employee satisfaction, performance and organizational commitment” (de Koster et al, 2011: pp. 77-78). We measure SSTL using the scale originally used by de Koster et al. (2011) consisting of 10 items, measuring all communicative sub-dimensions of SSTL through 7-point Likert scales ( $1=$ I totally disagree to $7=$ I totally agree). Cronbach's alpha for SSTL was good $\alpha=.94$.

Biographic Data. At the end of the questionnaire, respondents were asked to indicate their gender, age, education and nationality. To evaluate the general level of safety of Dutch warehouses compared to warehouses abroad, respondents were also asked to compare the safety of the company they work for in the Netherlands with the general level of safety of companies in their country of origin. Respondents were asked to complete the statement "In comparison with companies in my country of birth, this company is", using a 7-point Likert scale with 1 "less safe" and 7 "more safe". Next to that, respondents were asked to indicate whether they were born in the Netherlands or not. Within the last two general questions, respondents were asked to indicate which company they work for and to specify their working position, if they work as a warehouse employee, team leader or manager.

\subsection{Procedure and Respondents}

The present study was conducted among 224 workers in 13 randomly selected Dutch warehouses, the sectors ranged from the logistics sector to food supply warehouses. Warehouse employees were the largest respondents group with $82.1 \%$. The criterion for the selection of Dutch warehouses was a certain degree (at least 5\%) of internationality and cultural diversity among the workforce. Among the respondents, 133 (59.4\%) were born in the Netherlands and 87 (38.8\%) were not. Seven different nationalities were identified within the group of respondents, $67.4 \%$ were Dutch, .4\% English, 3.6\% German, 17.4\% Polish, 0.4\% Hungarian, .4\% Czech and 9.8\% had a different nationality. Looking at the educational level of respondents, most respondents had a degree in trade/technical/vocational training (41.1\%), $29.9 \%$ in high school, $19.2 \%$ in college or university and 5.8\% in 
primary school. Of the respondents, $82.6 \%$ were male and $15.2 \%$ female. The respondents mean age was 35 years. Respondents were between 16 and 61 years old.

Dutch warehouses were contacted via email or telephone and provided with a written or oral introduction that the research was about different leadership styles in multicultural teams and asked whether the workforce had a certain degree of internationality. After confirmation to conduct the research, respondents were asked to fill in a paper questionnaire that was provided by the researcher in person. Respondents could select between Dutch, English, German and Polish questionnaires. The questionnaire included 50 questions and a short instruction that the research is on different leadership styles, that all data will be treated anonymously, only serving the purpose of the research, and information on how to answer the questions. It took respondents about 5 minutes to fill in the survey. As the researchers were present during the answering procedure, respondents were allowed to ask if they had difficulties understanding the questionnaire. At the end of the questionnaire, respondents were thanked for their participation.

\section{Results}

\subsection{Cultural Values and Safety on the Workfloor}

First, a regression analysis was conducted to establish the role of uncertainty avoidance on safety awareness. Table 1 shows that the variable uncertainty avoidance explained $19 \%$ of the variance in safety awareness $(F$ $(2,220)=26.79, p<.001)$. Uncertainty avoidance was shown to be a significant predictor of safety awareness ( $\beta$ $=.43, p<.001$.

An independent sample t-test also showed a significant difference between safety awareness of high and low levels of Uncertainty Avoidance $(t(19.90)=3.27, p=.004)$. Respondents with a high level of uncertainty avoidance $(M=6.26, S D=.84)$ were shown to have a significantly higher safety awareness than respondents with a low level of uncertainty avoidance $(M=4.89, S D=1.85)$.

\subsection{Leadership Style and Fostering Safety at the Multicultural Workfloor}

To establish the role of employees' perceived level of SSTL as a predictor of safety awareness, one linear regression analyses was conducted. Table 2 summarizes the results and shows that the variable SSTL entered explained $9 \%$ of the variance in safety awareness $(F(1,220)=22.39, p<.001)$ and that SSTL was indeed found to be a significant predictor of safety awareness $(\beta=.30, p<.001)$.

To examine the differences in safety awareness between respondents that indicated high or low levels of SSTL, the variable SSTL was recoded into two different variables: low (values 1 - 3.99) and high (values 4.99 7) level of SSTL. An independent t-test showed a significant difference between safety awareness of respondents who indicated to perceive a high or low level of perceived SSTL $(t(50.34)=2.82, p=.007)$.

Respondents who indicated a high level of SSTL $(M=6.28, S D=.89)$ were shown to have a significantly higher level of safety awareness than respondents who indicated a low level of SSTL ( $M=5.62, S D=1.40)$.

Table 1. Lineair regression analysis for the cultural dimension uncertainty avoidance as a predictor of safety awareness $(N=$ 224).

\begin{tabular}{llcc}
\hline Safety awareness & $B$ & $S E$ & $\beta$ \\
Uncertainty avoidance & .36 & .05 & $.43^{* * *}$ \\
$R^{2}$ & .19 & & \\
$F$ & $26.79^{* * *}$ & \\
\hline
\end{tabular}

${ }^{* *} p<.0001{ }^{* * *} p<.001$.

Table 2. Linear regression analysis for SSTL as a predictor of safety awareness $(N=224)$.

\begin{tabular}{llcc}
\hline Safety awareness & $B$ & $S E$ & $\beta$ \\
SSTL & .24 & .05 & $.30 * *$ \\
$R^{2}$ & .09 & & \\
$F$ & $22.39 * *$ & & \\
$* * p<.001$. & &
\end{tabular}


SSTL Leadership Dimensions

A multiple regression analysis with the five dimensions of SSTL, outlined by Barling et al. (2002), as possible predictors of safety awareness was conducted to identify specific and more detailed leadership trades as possible predictors of safety awareness.

A multiple regression analysis with the five SSTL dimensions as possible predictive factors of safety awareness revealed that these dimensions explain $9 \%$ of the variances in safety awareness $(F(5,212)=5.27, p<.001)$. Table 3 summarizes these results and shows that Idealized Influence was found to be a significant predictor of safety awareness $(\beta=.26, p=.032)$. Inspirational Influence $(\beta=-.03, p=.830)$, Intellectual Stimulation ( $\beta$ $=.12, p=.421)$, Individual Consideration $(\beta=.03, p=.838)$ and Contingent Reward $(\beta=.18, p=.840)$ were not found to be significant predictors.

After categorizing the variable Idealized Influence into high and low values, an independent t-test also showed a difference between high and low Idealized Influence and safety awareness $(t(189)=4.38, p<.001)$. Respondents who rated their leader high on Idealized Influence $(M=6.22, S D=.91)$ were shown to have a higher safety awareness than respondents who rated their leader low on Idealized Influence $(M=5.30, S D=$ 1.62).

\section{Discussion \& Conclusion}

In the light of a culturally diverse workforce and the search for successful safety and leadership strategies that enhance a safe work environment, the purpose of this study was to investigate which factors predict a better safety climate. In particular, different levels of uncertainty avoidance as well as of Safety-Specific Transformational Leadership were studied as potential predictors of safety awareness and the two underlying constructs, safety performance and awareness, within Dutch warehouses.

High Uncertainty Avoidance is associated with preventing unpredictable and ambiguous situations and relying on fixed and clear regulations (Hofstede, 2001). Therefore, comparing these seemingly cautious characteristics to workplace' safety behaviour, we expected high uncertainty avoidance to predict a better safety climate. The findings are in line with these expectations. We indeed find that safety awareness is not only predicted by uncertainty avoidance but it appears that people with higher levels of uncertainty avoidance are also more aware of safety and also have a better overall perception of the "organizational approaches to safety" (Barling et al., 2002). These findings are in line with the results of Mohamed \& Yule (2009) and contrary to Reniers \& Gidron (2013) who did not find a significant relationship between uncertainty avoidance and occupational safety. Workers with a lower level of uncertainty avoidance were shown to be less aware of workplace safety and also rated the safety climate within their organization lower. Safety programs should therefore pay additional attention to create safety awareness in people with low levels of uncertainty avoidance. As the present study shown, leadership style can be an important tool in predicting and motivating safety awareness.

In line with Barling et al. (2002) and de Koster et al. (2011), it seems that safety specific transformational leadership is indeed a leadership style that enhances safety awareness. Additionally, we find that when employees perceive their leader's style as more SSTL, this then indeed enhances safety awareness. SSTL, a style

Table 3. Multiple regression analysis for the different dimensions of SSTL that predict safety awareness $(\mathrm{N}=224)$.

\begin{tabular}{llll}
\hline Safety awareness & $B$ & $S$ & $\beta$ \\
\hline Idealized influence & .19 & .09 & $.26^{* *}$ \\
Inspirational influence & -.02 & .11 & -.03 \\
Intellectual stimulation & .08 & .10 & .12 \\
Individual consideration & .02 & .10 & .03 \\
Contingent reward & -.01 & .05 & .18 \\
$R^{2}$ & .09 & & \\
$F$ & $5.27^{* * *}$ & & \\
\hline
\end{tabular}

$* * p<.05 * * * p<.001$. 
that focuses on personal commitment and encourages employees to reflect on safety values (de Koster et al., 2011) is also found to predict safety climate, the overall perception of the organizational safety environment (Barling et al., 2002). In line with this prior research, we originally assumed that only SSTL would have this positive predictive role for safety awareness. However, it appears that transactional leadership also predicts safety performance and awareness.

Looking at the five specific communicative SSTL characteristics, outlined by Barling et al. (2002), this study has taken a step in identifying specific communicative leadership skills that can enhance employees' safety awareness. Idealized influence predicted safety awareness significantly. When employees perceived their leader to have idealized influence, their safety awareness was higher, as well. Showing commitment to safety values and being perceived as a leader who is personally engaged in creating a safe work environment, expressing own responsibility for occupational safety and encouraging employees to think can create more safety awareness among the workforce (Barling et al., 2002). In line with Barling et al. (2002), we find that when leaders show idealized influence, safety awareness is enhanced through "commitment rather than obligation to comply" (Barling et al., 2002: p. 489). Safety programs for organizations should therefore not only focus on mandatory rules and regulations but also on the idealized influence of the leader. Clearly, the findings of the present study indicate that taking into account cultural differences and the appropriate leadership style is crucial to enhance safety climate.

\section{Acknowledgements}

I would like to thank all my Bachelor-3 students (cohort 2014-1015) in Communication and Information Studies at Radboud University, The Netherlands for helping with collecting all the data.

\section{References}

Barling, J., Weber, T., \& Kelloway, T. K. (1996). Effects of Transformational Leadership Training on Attitudinal and Financial Outcomes: A Field Experiment. Journal of Applied Psychology, 81, 827-832. http://dx.doi.org/10.1037/0021-9010.81.6.827

Barling, J., Loughlin, C., \& Kelloway, E. (2002). Development and Test of a Model linking Safety Specific Transformational Leadership and Occupational Safety. Journal of Applied Psychology, 81, 488-496. http://dx.doi.org/10.1037/0021-9010.87.3.488

Culpepper, R. A., \& Watts, L. (1999). Measuring Cultural Dimensions at the Individual Level: An Examination of the Dorfman and Howell (1988) Scales and Robertson and Hoffman (1999) Scale. Academy of Strategic and Organizational Leadership Journal, 3, 22-34.

Hale, A. R., Guldenmund, F. W., Van Loenhout, P. L. C. H., \& Oh, J. I. H. (2010). Evaluating Safety Management and Culture Interventions to Improve Safety: Effective Intervention Strategies. Safety Science, 48, 1026-1035. http://dx.doi.org/10.1016/j.ssci.2009.05.006

Hofstede, G. (2011). Dimensionalizing Cultures: The Hofstede Model in Context. Online Readings in Psychology and Culture, 2, 8. http://dx.doi.org/10.9707/2307-0919.1014

Kearney, E., \& Gebert, D. (2009). Managing Diversity and Enhancing Team Outcomes: The Promise of Transformational Leadership. Journal of Applied Psychology, 94, 77-89. http://dx.doi.org/10.1037/a0013077

Kelloway, E. K., Mullen, J., \& Francis, L. (2006). Divergent Effects of Transformational and Passive Leadership on Employee Safety. Journal of Occupational Health Psychology, 11, 76-86. http://dx.doi.org/10.1037/1076-8998.11.1.76

de Koster, R. B. M., Stam, D., \& Balk, B. M. (2011). Accidents Happen: The Influence of Safety-Specific Transformational Leadership, Safety Consciousness, and Hazard Reducing Systems on Warehouse Accidents. Journal of Operations Management, 29, 753-765. http://dx.doi.org/10.1016/j.jom.2011.06.005

Mearns, K., \& Yule, S. (2008). The Role of National Culture in determining Safety Performance: Challenges for the Global Oil and Gas Industry. Safety Science, 47, 777-785. http://dx.doi.org/10.1016/j.ssci.2008.01.009

Mohamed, K., \& Yule, S. (2009). National Culture and Safe Work Behaviour of Construction Workers in Pakistan. Safety Science, 47, 29-35. http://dx.doi.org/10.1016/j.ssci.2008.01.003

Reniers, G., \& Gidron, Y. (2013). Do Cultural Dimensions predict Prevalence of Fatal Work Injuries in Europe? Safety Science, 58, 76-80. http://dx.doi.org/10.1016/j.ssci.2013.03.015

Saee, J. (2005). Managing Organizations in a Global Economy. An Intercultural Perspective. United States of America: Thomson, South-Western. 


\section{Submit or recommend next manuscript to SCIRP and we will provide best service for you:}

Accepting pre-submission inquiries through Email, Facebook, Linkedin, Twitter, etc A wide selection of journals (inclusive of 9 subjects, more than 200 journals)

Providing a 24-hour high-quality service

User-friendly online submission system

Fair and swift peer-review system

Efficient typesetting and proofreading procedure

Display of the result of downloads and visits, as well as the number of cited articles

Maximum dissemination of your research work

Submit your manuscript at: http://papersubmission.scirp.org/ 